\title{
Novel continuum modeling of crystal surface evolution
}

\author{
Navot Israeli* and Daniel Kande $* *$ \\ Department of Physics of Complex Systems, Weizmann Institute of Science, Rehovot 76100, Israel
}

\begin{abstract}
We propose a novel approach to continuum modeling of the dynamics of crystal surfaces. Our model follows the evolution of an ensemble of step configurations, which are consistent with the macroscopic surface profile. Contrary to the usual approach where the continuum limit is achieved when typical surface features consist of many steps, our continuum limit is approached when the number of step configurations of the ensemble is very large. The model can handle singular surface structures such as corners and facets. It has a clear computational advantage over discrete models.
\end{abstract}

PACS numbers: 68.55.-a, 68.35.Ja

The behavior of classical physical systems is typically described in terms of equations of motion for discrete microscopic objects (e.g. atoms). In many cases, the behavior of such systems is smooth when observed on macroscopic length and time scales. It is useful to describe these systems in terms of continuum, coarsegrained models, which treat the dynamics of the macroscopic, smoothly varying, degrees of freedom rather than the microscopic ones. Such models are more amenable to analytical treatments and have enormous computational advantages over their discrete counterparts.

Many physical systems exhibit a macroscopically smooth behavior everywhere, except in small regions of space where their behavior is singular. Examples are dislocations in a crystal, cracks in crystalline material, facet edges on crystal surfaces, etc. These singular regions are of interest because they frequently drive the dynamics of the whole system. It is an interesting and important challenge to develop continuum descriptions of such systems, since standard phenomenological continuum models completely fail in the singular regions.

In this Letter we address the above problem in the context of the dynamics of crystal surfaces. Below the roughening temperature the evolution of these surfaces proceeds by the nucleation, flow and annihilation of atomic steps. These steps originate either from a miscut of the initial surface with respect to a high symmetry plane of the crystal or as the boundaries of islands and vacancies which nucleate on the surface during morphological evolution (we ignore screw dislocations as sources of steps). The steps are separated by terraces, which are parallel to a high symmetry crystal orientation. Each step consists of atomic kinks separated by straight portions along closed-packed orientations. However, on length scales larger than the typical distance between kinks the step is a smooth line. Thus the evolution of the surface corresponds to the motion of discrete smooth lines.

One can model step motion by solving the diffusion problem of adatoms on the terraces with boundary conditions at step edges. These conditions account for attachment and detachment of atoms to and from step edges. The local flux of adatoms at step edges and the resulting step velocities can then be calculated. This approach was introduced long ago by Burton, Cabrera and Frank [1], and was further developed by other authors [2]. Given the arrangement of few neighboring steps, these models allow a calculation of the normal velocity of a step. The number $M$ of neighboring steps on each side required for this calculation depends on the step kinetics and on the interaction range between steps (in the examples below $M=2$ ). Step models are capable of describing surface evolution on the mesoscopic scale with significant success 3, 1. Such models pose a serious challenge for numerical computations, and can be solved only for small systems.

Several attempts were made to construct continuum models of stepped surfaces [5 17], to study their large scale properties. The general idea behind these attempts is that step flow can be treated continuously in regions where every morphological surface feature is composed of many steps. If we label surface steps by the index $n$, the continuum limit in these models is obtained by taking $n$ to be continuous. The outcome of these attempts are partial differential equations for surface evolution. Such continuum models are fairly successful in describing the evolution of smooth surfaces of very simple morphology. However, below the roughening temperature, crystal surfaces have singularities in the form of corners and macroscopic facets. The latter are a manifestation of the cusp singularity of the surface free energy at high symmetry crystal orientations. The assumption that every surface feature is composed of many steps clearly breaks down on macroscopic facets where there are no steps at all. Thus, existing continuum models fail near singular regions.

A possible resolution of this problem is to solve the continuum model in non-singular regions and use matching conditions at the singular facet edges 11 14]. This is a good solution in extremely simple cases (see, e.g. [14]), but generally it is not possible to derive the appropriate matching conditions from the microscopic models [12,13]. As a result this approach is not very useful.

Another suggested solution is to round the surface free energy cusp 15 17]. The resulting continuum models replace facets by relatively flat but analytic regions. As explained below, this approach does not correctly reproduce the evolution of facet edges. Thus, all existing continuum models fail in the description of the evolution of 
surfaces with singularities. This failure is both conceptual and practical, in the sense that it leads to qualitatively as well as quantitatively incorrect results.

We now propose a conceptually new definition of the continuum limit, which we term configurational continuum. This definition allows construction of continuum models, free of all the limitations of standard continuum models. Our key observation is that a continuous surface height profile can be represented by many similar, but not identical, step configurations. Figure 1 is a demonstration of this point for a one dimensional surface. It shows a continuous height function, $h(x)$, as a function of position $x$ (thick solid line), and three valid discrete representations of this profile as step configurations. In configurational continuum we define the continuous height profile as the upper envelope of the discrete height functions of an ensemble of many such step configurations. To construct these configurations, let $a$ be the height of a single step and $N$ the number of configurations in the ensemble. We construct the ensemble so that the height difference $\delta h$, between two adjacent configurations is $a / N$, as depicted in Fig. 1. The configurational continuum limit is obtained when $N \rightarrow \infty$. The generalization to higher dimensions is straightforward.

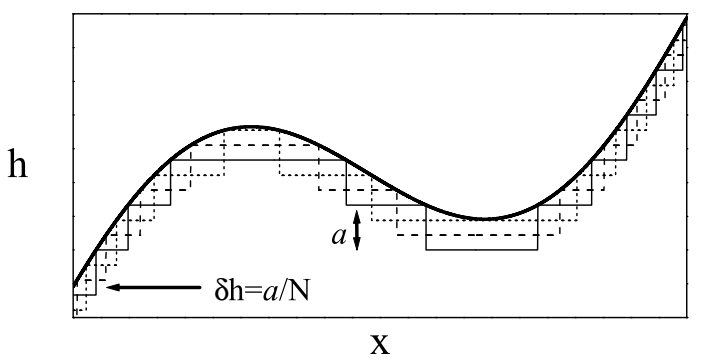

FIG. 1. A schematic illustration of the ensemble of configurations whose upper envelope defines the configurational continuum limit.

The dynamics of the continuum model is as follows. Each step configuration of the ensemble evolves according to the microscopic dynamics. As a result, the envelope of discrete height functions changes with time, thus defining the evolution of the continuous height function.

The crucial assumption hidden in this definition of the continuum model is that our construction leads to a mathematically well-defined height function at all times. When does this assumption hold? Consider two initially similar configurations of the ensemble. Our continuum limit is well-defined provided these two configurations have similar microscopic dynamics and hence remain similar at later times [18. This assumption has to hold in the standard continuum definition as well. If two initially similar configurations evolve very differently, one must follow the specific microscopic configuration of interest.

We now derive the evolution equation for the contin- uous height of a two dimensional surface, $h(\vec{r}, t)$, at position $\vec{r}$ and time $t$. Here $\vec{r}$ is a vector in the $x y$ plane, defined as the high symmetry crystal plane parallel to the terraces. $h$ changes with time due to the flow of steps through $\vec{r}$, and due to nucleation and annihilation of steps. At this stage we disregard nucleation processes and include them later. First, we consider positions, which are not local extrema of the height profile. It is obvious from the construction of the configurational continuum, that for each point $\vec{r}$ there is exactly one configuration in the ensemble, which has a step that passes through $\vec{r}$ at the time $t$. That step lies along the unique equal-height contour line, which passes through $\vec{r}$. As is demonstrated in Fig. 1, the exact positions of neighboring steps in the same configuration can be calculated from the knowledge of $h(\vec{r}, t)$, and the fact that in this configuration there is a step at $\vec{r}$. Hence, we can use the discrete step model to calculate the normal velocity of the step at position $\vec{r}$ at time $t$. Let us denote this velocity by $\vec{v}_{s}(\vec{r}, t)$. Note that at different positions, $\vec{v}_{s}$ is the normal velocity of steps which belong to different configurations in the ensemble.

Next we define a two dimensional unit-vector $\hat{n}(\vec{r}, t)$ in the $x y$ plane, which is normal to the contour line at $\vec{r}$, and points in the direction opposite to $\vec{v}_{s}(\vec{r}, t)$. This allows us to define the directional gradient

$$
\nabla h_{\hat{n}}(\vec{r}, t) \equiv \hat{n}(\vec{r}, t) \lim _{\epsilon \rightarrow 0_{+}} \frac{h(\vec{r}+\epsilon \hat{n}, t)-h(\vec{r}, t)}{\epsilon},
$$

where the directionality is needed in order to deal with corners and facet edges. The gradient is in the direction from which steps flow towards the point $\vec{r}$. This is useful for the calculation of the current of steps arriving at $\vec{r}$ :

$$
J(\vec{r}, t)=\frac{N}{a}\left|\nabla h_{\hat{n}}(\vec{r}, t) \cdot \vec{v}_{s}(\vec{r}, t)\right|,
$$

where we have used the fact that the local step density is $\left|\nabla h_{\hat{n}}(\vec{r}, t)\right| N / a$. Note that $J$ is the current of steps belonging to all configurations in the ensemble, and not to one particular configuration. Since each step (from any configuration), which passes through $\vec{r}$ changes the height of the ensemble envelope by $a / N$ (see Fig. 11), the continuous height profile obeys the evolution equation

$$
\frac{\partial h(\vec{r}, t)}{\partial t}=-\nabla h_{\hat{n}}(\vec{r}, t) \cdot \vec{v}_{s}(\vec{r}, t)
$$

The above derivation of the evolution in the continuum is not valid at local extrema of the surface, because generally one cannot define a unique equal-height contour line which passes through such a point. To avoid the problem, we define $\partial h / \partial t$ at local extrema as the limit of the height time derivative as one approaches these points. This is justified, since there are no microscopic realizations of the surface with steps exactly at the local extrema.

At this point we emphasize that the configurational continuum evolution is formally identical to the evolution of the discrete step model. This statement is almost 
trivial, since by definition Eq. (3) follows the envelope defined by the ensemble of configurations, and each configuration evolves with step velocities calculated from the discrete step model. Thus Eq. (3) is exact. Similarly to other continuum models it is solved numerically by discretization of space, which is the only approximation involved in such solutions.
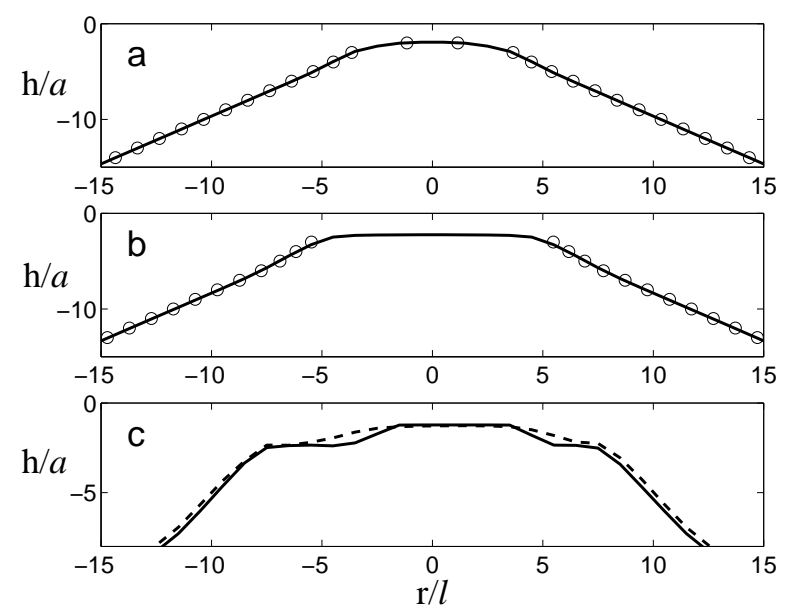

FIG. 2. Evolution of a crystalline cone with growth flux. The height (in units of $a$ ) is shown vs. the radius (in units of the initial terrace width, $l$ ). The lines show cross sections of the two dimensional solution of Eq. (3). Circles show the surface evolution according to the one dimensional step flow model. The initial shape is an exact cone, and (a) and (b) show the generation and evolution of a facet at the top at later times. (c) shows an island which nucleated (solid) and grew (dashed) on the top facet.

What is the relation between configurational continuum and other existing continuum models? We will show elsewhere that in regions where $h(\vec{r}, t)$ is a smooth function of $\vec{r}$, the evolution equation (3) reduces to the differential equation of standard continuum models. However, near corners or facets (3) accounts for the discrete nature of steps and cannot be written as a differential equation, due to the singular nature of the profile. Contrary to other continuum models, configurational continuum is exact even at singular points and is capable of describing both smooth and singular behaviors without artificial smoothing or matching conditions. To the best of our knowledge, configurational continuum is the only continuum model capable of correctly treating the dynamics of singular lines [19].

The fact that no matching conditions are needed in configurational continuum is truly important for possible generalizations. A matching condition approach has been used in other areas, such as plasticity of crystals. The idea there is to solve continuum elasticity far from dislocations and a discrete model near dislocations. The inaccurate matching conditions between the different regions lead in this case to artificial reflection of phonons from the boundaries between the regions [20]. A proper generalization of configurational continuum to atomistic systems may eliminate these artificial phonon reflections.

In terms of computational efficiency, our model is comparable to standard continuum models. The computational advantage over discrete models arises from the fact that in macroscopically smooth regions the density of steps is much larger than the needed density of discretization points. Some care should be taken near singular lines, and discretization there should be dense enough to allow an accurate interpolation of the singular profile. This, however, should not affect the overall efficiency, and when most of the surface is smooth the number of steps needed to be simulated is usually orders of magnitude smaller than the number of steps in the system.

We now apply the configurational continuum approach to a few simple cases in order to demonstrate its validity. To this end, we consider the evolution of a conic structure which consists of circular concentric steps. The adatom concentration on the terraces can be integrated out due to the radial symmetry of the system, yielding a set of coupled non-linear differential equations for the evolution of step radii [13]. We have solved this discrete model numerically with and without growth flux. In the absence of flux, the cone decays by step flow. During the decay, a facet develops at the top of the cone, and its radius grows as $t^{1 / 4}$. With flux, the cone grows except at the peak which initially decays and then saturates. A facet forms at the peak after saturation.

The continuum model we solved for this example is a two dimensional model, which can, in principle, develop non radially symmetric morphology. The microscopic dynamics we used was a two dimensional generalization of the microscopic equations for step radii of the discrete one dimensional model. Any microscopic dynamics, such as a full solution of the diffusion equation on each terrace, can be used in the framework of configurational continuum. For the sake of demonstrating the validity of our approach the simple dynamics we chose is sufficient.

Figures 2(a) and (b) show a comparison between a numerical solution of the discrete step model with growth flux and a cross section of the two dimensional surface obtained from the solution of our continuum model at various times. The agreement is quite impressive. In particular, the width and height of the facet obtained from the continuum model are equal (within numerical accuracy) to those of the discrete model at all times. A similar agreement between the two models is achieved in the absence of growth flux. For comparison, we also investigated the dynamics of the decaying cone using the continuum model of 17]. Within this model, the cusp in the free energy is rounded, and the evolution is studied with various degrees of rounding. We found that even in the limit of small rounding the height profile is very different from the profile of the discrete model. Although a facet does develop, its size is about $40 \%$ larger than the facet of the discrete model at all times. In addition, the 
shapes of the height profile differ qualitatively.

Island nucleation can also be included in our continuum model. To this end we add a microscopic nucleation scheme, which defines the nucleation probability at any given position on the surface, given a specific microscopic step configuration. Within our continuum approach, the nucleation probability at a point on the continuous surface is the ensemble average of the microscopic nucleation probabilities at this point. For demonstration purposes we included the simple scheme where the nucleation probability in any given step configuration is proportional to the square of the local adatom concentration. In fact, island nucleation is already included in the simulations presented in Fig. 2. Nucleation events occur on the top facet once it becomes large enough, but there is hardly any nucleation on the finite slope regions, because the terraces there are very narrow. Figure 2(c) shows an island on the top facet, which nucleated and started growing. Vacancy nucleation during evaporation can be included in a similar manner.

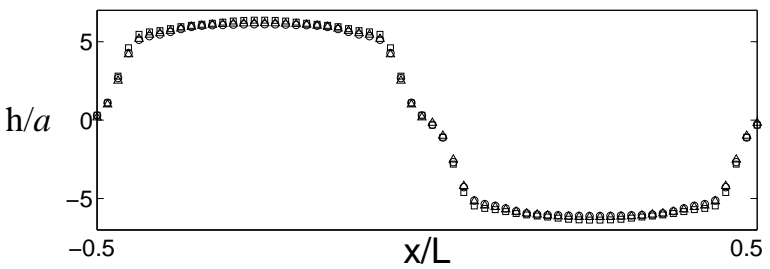

FIG. 3. Data collapse for week repulsive interactions between steps. Shown are cross sections (along the $x$ direction at $y=-L / 4$ ) of bidirectional sinusoidal profiles with different wave lengths after a relaxation time of $t_{0} L^{3}$. Wave lengths shown are $L=64$ (circles), 128 (squares) and 256 (triangles).

We now turn to the more demanding example of bidirectional sinusoidal grating relaxation. Here the initial surface height profile of wave length $L$ is given by $h_{L}(\vec{r}, t=0)=h_{0} \sin (2 \pi x / L) \sin (2 \pi y / L)$. The relaxation of this profile towards a flat surface was studied by Rettori and Villain [21], who gave an approximate solution to a step flow model, in the limit where the interaction between steps can be neglected in comparison with the step line tension. The decay of bidirectional sinusoidal profiles was also studied numerically [17]. We now apply our model to this problem assuming diffusion limited kinetics without deposition flux.

For weak interactions between steps, the surface height evolves according to $\partial h_{L} / \partial t \propto \nabla^{2} \kappa \sim L^{-3}$, where $\kappa$ is the step curvature. We therefore expect the following scaling law: $h_{L}(\vec{r}, t)=h_{L=1}\left(\vec{r} / L, t / L^{3}\right)$. Figure 3 shows the data collapse of cross sections of profiles from the configurational continuum model. The different symbols correspond to different wave lengths at time $t=t_{0} L^{3}$ for a fixed $t_{0}$. The quality of the data collapse shows that the scaling scenario holds very accurately. Note that large facets have developed at the surface extrema, and they are connected by very steep slopes. This shape does not agree with Rettori and Villain's heuristic argument [21], which predicts that facets appear also near $h=0$ lines. Nevertheless, their prediction that the decay time of the height profile goes as $L^{3}$ is in agreement with both the scaling law and our numerical solutions. Our simulations show that for much stronger repulsive interactions between steps, the scaling law does not hold.

* navot@uiuc.edu

** daniel.kandel@weizmann.ac.il, http://www.weizmann.ac.il/ fekande

[1] W. K. Burton, N. Cabrera and F. C. Frank, Philos. Trans. R. Soc. London, Ser. A 243, 299 (1951).

[2] For general reviews see H.-C. Jeong and E. D. Williams, Surf. Sci. Rep. 34, 175 (1999); E. D. Williams, Surf. Sci. 299/300, 502 (1994).

[3] E. S. Fu, M. D. Johnson, D.-J. Liu, J. D. Weeks and E. D. Williams, Phys. Rev. Lett. 77, 1091 (1996).

[4] S. Tanaka, N. C. Bartelt, C. C. Umbach, R. M. Tromp and J. M. Blakely, Phys. Rev. Lett. 78, 3342 (1997).

[5] W. W. Mullins, J. Appl. Phys. 28, 333 (1957).

[6] M. Ozdemir and A. Zangwill, Phys. Rev. B 42, 5013 (1990).

[7] P. Nozières, J. Phys. I France 48, 1605 (1987).

[8] F. Lançon and J. Villain, Phys. Rev. Lett. 64, 293 (1990).

[9] M. Uwaha, J. Phys. Soc. Jap. 57, 1681 (1987).

[10] A. Chame, S. Rousset, H. P. Bonzel and J. Villain, Bul. Chem. Commun. 29, 398 (1996/1997).

[11] J. Hager and H. Spohn, Surf. Sci. 324, 365 (1995).

[12] N. Israeli and D. Kandel, Phys. Rev. B 6213707 (2000).

[13] N. Israeli and D. Kandel, Phys. Rev. Lett. 803300 (1998); Phys. Rev. B 605946 (1999).

[14] N. Israeli, H.-C. Jeong, D. Kandel and J. D. Weeks, Phys. Rev. B 615698 (2000).

[15] H. P. Bonzel, E. Preuss and B. Steffen, Appl. Phys. A 35, 1 (1984).

[16] H. P. Bonzel and E. Preuss, Surf. Sci. 336, 209 (1995).

[17] M. V. Ramana Murty, Phys. Rev. B 62, 17004 (2000).

[18] Note that the height is not well-defined if overhangs are generated. In this case it is possible to generalize the continuous function to appropriately describe the overhangs.

[19] We consider the level set method (see, e.g. M. F. Gyure et al., Phys. Rev. E 58, 6927 (1998)) to be a discrete model in this context. While it uses a continuous function in order to follow the evolution of the surface, this function is only a mathematical tool, which traces the evolution of all steps in the system as discrete objects. The level set method is perhaps the most efficient discrete step model, but its efficiency is far from that of continuum models for macroscopically smooth surfaces.

[20] See, e.g., W. Cai, M. de Koning, V. V. Bulatov and S. Yip, Phys. Rev. Lett. 85, 3213 (2000).

[21] A. Rettori and J. Villain, J. Phys. France 49, 257 (1988). 